\title{
Computational Modeling of Meteor-Generated Ground Pressure Signatures
}

\author{
Marian Nemec* and Michael J. Aftosmis ${ }^{\dagger}$ \\ NASA Ames Research Center, Moffett Field, CA 94035, USA \\ Peter G. Brown ${ }^{\ddagger}$ \\ University of Western Ontario, ON, N6A 3K\%, Canada
}

\begin{abstract}
We present a thorough validation of a computational approach to predict infrasonic signatures of centimeter-sized meteoroids. We assume that the energy deposition along the meteor trail is dominated by atmospheric drag and simulate the steady, inviscid flow of air in thermochemical equilibrium to compute the meteoroid's near-body pressure signature. This signature is then propagated through a stratified and windy atmosphere to the ground using a methodology adapted from aircraft sonic-boom analysis. An assessment of the numerical accuracy of the nearfield and the farfield solver is presented. The results show that when the source of the signature is the cylindrical Mach-cone, the simulations closely match the observations. The prediction of the shock rise-time, the zero-peak amplitude of the waveform, and the duration of the positive pressure phase are consistently within $10 \%$ of the measurements. Uncertainty in the shape of the meteoroid results in a poorer prediction of the trailing part of the waveform. Overall, our results independently verify energy deposition estimates deduced from optical observations.
\end{abstract}

\section{Introduction}

$\mathrm{T}$ he hypersonic entry of meteoroids into the atmosphere generates strong shock waves that decay as they propagate to the ground. Delayed sounds from meteors have been known since ancient times. Systematic investigations of meteoric pressure disturbances began in the early part of the $20^{\text {th }}$ century, even being proposed as an early form of atmospheric temperature probing ${ }^{1}$ Nowadays, pressure signatures recorded by dedicated sensor arrays are being actively investigated to explain various aspects of meteoroid entry, impact and origin.2 ${ }^{2}$ 4

Along with observational capabilities, analytic models of meteor-generated pressure waves were developed by Tsikulin ${ }^{5}$ and ReVelle $\sqrt[6]{6}$ These models provide a simple prediction of the period and maximum amplitude of the wave when it reaches the ground. Near the trajectory, the strong bow shock of the meteoroid is modeled as a cylindrical blast wave that decays into a weak shock called an $\mathrm{N}$-wave. The attenuation of the $\mathrm{N}$-wave as it propagates through the atmosphere is approximated using weak-shock theory and, as the effects of nonlinearity diminish, linear wave theory. ReVelle 6 [ 7 derived closed-form expressions for the period and maximum amplitude from relations involving the blast-wave radius, geometrical acoustics and empirical correlations based on studies of lightning discharges 8

The analytic models indicate that the detection of meteoric infrasound ${ }^{\circledR}$ should be fairly common especially infrasound from small regional events where the wave propagation distance through the atmosphere is relatively short. Until recently, however, few such measurements existed ${ }^{[4}$ Consequently, the models have not been properly validated despite being widely employed in both the infrasound and meteor communities to estimate energy deposition $10[11$

\footnotetext{
*Aerospace Engineer, Computational Aerosciences Branch, MS 258-5; marian.nemec@nasa.gov. Senior Member AIAA.

†Aerospace Engineer, Computational Aerosciences Branch; michael.aftosmis@nasa.gov. Associate Fellow AIAA.

¥Professor, Department of Physics and Astronomy, pbrown@uwo.ca.

${ }^{\text {a }}$ Inaudible, low-frequency $(<20 \mathrm{~Hz})$ pressure waves.
} 
To address this issue, a long-term campaign of simultaneous optical and infrasound measurements began in 2006 in Canada, with the goal of producing a well-constrained meteor dataset that includes orbital and trajectory parameters, light curves, and pressure signatures 12,13 The instrumentation involves an optical network of $7-14$ all-sky camera stations that detects and astrometrically measures meteors. ${ }^{14}$ The pressure signatures are recorded at the Elginfield Infrasound Array (ELFO), which is a four-sensor microbarograph array located near the geographical center of the optical network. Observations through the end of 2011 captured 71 events. ${ }^{13}$ These were analyzed by Silber et al., ${ }^{15}$ who found that the analytic model of ReVelle ${ }^{7}$ produces reasonable agreement with optical observations when the meteoroid's blast radius is estimated from period measurements. Comparisons based on amplitude measurements, however, show a large mismatch, hinting at shortcomings of the ReVelle model ${ }^{7}$ that Silber et al ${ }^{15}$ attribute chiefly to the choice of the transition altitude from the weakly-nonlinear to the linear regime.

To improve the prediction of the ground waveform, Haynes and Millet ${ }^{16}$ modify the ReVelle model ${ }^{17}$ by implementing the F-function approach of Whitham ${ }^{17}$ for the propagation of weak shocks. This relaxes the requirement of explicitly specifying the transition altitude. Henneton et al 18 explore a numerical approach that is much more general. They use computational fluid dynamics to determine the near-body flow around the meteoroid, extract a nearfield pressure signature, and then propagate this signature to the ground by solving the augmented Burgers' equation to accurately model atmospheric absorption. Their numerical experiments agree well with the ReVelle model ${ }^{7}$ in the asymptotic limits of infinite meteoroid velocity and vanishing size. Both Haynes and Millet ${ }^{16}$ and Henneton et al., $\frac{18}{18}$ however, test their simulations by reproducing the signature from the Carancas fireball-crater event, where details of the trajectory, energy and dynamics are very uncertain $19 \sqrt{20}$

This paper investigates a computational approach based on our work in simulation-based aircraft sonicboom prediction. ${ }^{21}$ The approach is similar to that of Henneton et al ${ }^{[18}$ We use the new, homogenous dataset of Silber et al ${ }^{15}$ to test our simulations. This dataset provides significantly better validation benchmarks than the Carancas event used in previous studies. The entry of the Stardust capsule, 22,23 which is a convenient "artificial" meteor with a known shape, is used for initial validation. Thereafter, we consider several wellconstrained meteor events from Silber et al.,$\frac{15}{15}$ which we previously examined in Nemec et al., $\frac{24}{24}$ and assess the ability of the computational approach to reproduce the observed waveforms. We provide details on the problem setup, and focus on the convergence characteristics and performance of the nearfield and farfield solvers to obtain accurate ground signatures.

More broadly, we demonstrate the potential of using high-fidelity simulations to interpret meteor observations. Our approach is especially valuable as an independent estimate of energy deposition, $\mathrm{d} E / \mathrm{d} \ell$, for meteoroids that generate observable ground-pressure signatures. Unlike previous analyses 415 that rely on the concept of blast radius to estimate $\mathrm{d} E / \mathrm{d} \ell$, we show that high-fidelity nearfield simulations, such as those of Henneton et al ${ }^{[18}$ and Aftosmis et al. ${ }^{[25}$ can be used to estimate energy deposition directly. Furthermore, accurate prediction of meteoric pressure signatures may help in calibrating, or anchoring, optical and radar observations, as well as improve the reconstruction of meteor events from solely infrasound observations.

\section{Problem Setup and Numerical Method}

Figure 1 shows a sketch of the physical domain with a meteoroid entering the atmosphere and generating a strong, detached shock wave. Our goal is to determine the ground pressure signature. We assume that the energy deposition, $\mathrm{d} E / \mathrm{d} \ell$, is wholly from the atmospheric drag force. This neglects energy loss due to radiation, but this component typically does not exceed $5 \%$ of the total $[26] 27$ A representative case is a small meteoroid with a characteristic diameter, $D$, not exceeding a few centimeters entering the upper atmosphere at a hypersonic speed of around $20 \mathrm{~km} / \mathrm{s}$. As shown in Figure 1, the meteoroid's Mach cone is essentially cylindrical, which is a consequence of the small Mach angle $\left(<2^{\circ}\right)$ due to the high entry speed.

Following the approach of Tsikulin ${ }^{5}$ and ReVelle $6 \sqrt{7}$ the physical domain is divided into a cylindrical nearfield region surrounding the trajectory and a farfield region, as illustrated in Figure 1. In the nearfield, close to the body, the flow is three-dimensional, strongly nonlinear, and dominated by complex physical processes that include real-gas effects, ablation, and often fragmentation. The use of computational fluid dynamics in this region avoids many of the simplifying assumptions that limit the accuracy of blast-wave theory, but presumes some knowledge of the energy deposition. As the bow shock propagates outward, the radial extent of the nearfield must be large enough to allow the shock to weaken sufficiently and the flow to become locally axisymmetric. The problem in the farfield can then be treated as a quasi-one-dimensional 


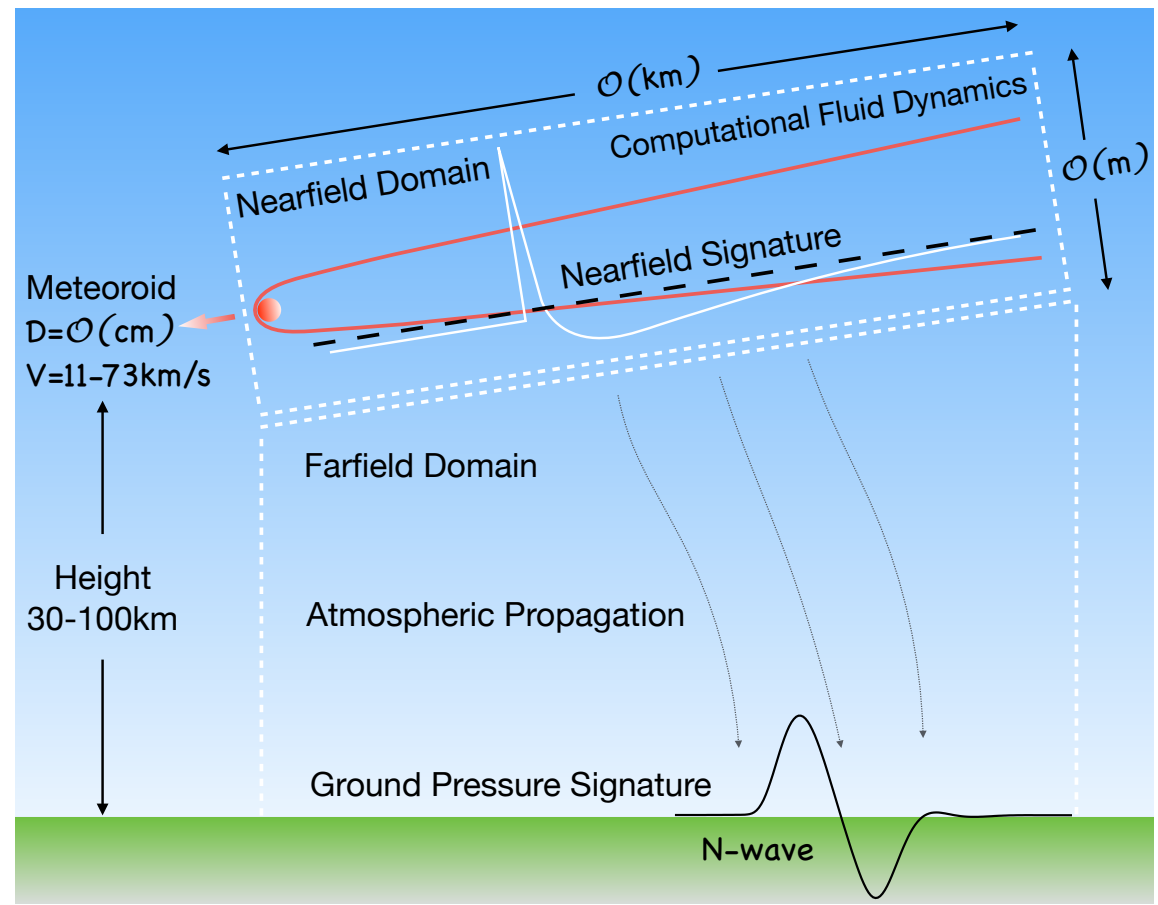

Figure 1. Sketch of meteoroid entry generating a strong, cylindrical, blast-like pressure pulse that attenuates as it propagates to ground.

propagation of an acoustic waveform. Figure 1 shows a typical, blast-like, nearfield signature (white line) that is extracted several body lengths below the trajectory of the meteoroid, along the black dashed line.

The ideas of splitting the physical domain and using a numerical simulation in the nearfield to initialize an atmospheric propagation in the farfield are well established in the field of aircraft sonic-boom analysis $17 \sqrt{28} \sqrt[31]{31}$ Moreover, simulation-based sonic-boom prediction has been thoroughly validated ${ }^{32}[33$ and is being applied regularly in the design of low-boom aircraft prototypes 34 In principle, it is straightforward to apply the computational approach of sonic-boom analysis to the prediction of meteoric pressure signatures. In practice, however, the challenges of hypersonic flow, longer and higher altitude atmospheric propagation, and weaker and lower-frequency signatures require special attention. In the next two subsections, we provide details of the nearfield and farfield solvers that specifically address these challenges.

\section{A. Nearfield Domain}

In the nearfield, we specify a uniform atmosphere and solve the steady, three-dimensional Euler equations governing the compressible flow of air in thermochemical equilibrium. Assuming negligible influence of ablation and a continuous medium, this inviscid flow model should predict nearfield signatures accurately for single-body meteoroids, except for viscous effects from the wake region. While not negligible, the pressure fluctuations in the wake due to viscous effects should be small relative to the large overpressure of the detached bow shock that dominates the signature.

For a finite region of space with volume $V$ and surface area $A$, the integral form of the Euler equations is given by

$$
\frac{\mathrm{d}}{\mathrm{d} t} \int_{V} \mathbf{Q} \mathrm{d} V+\oint_{A} \mathbf{F} \cdot \hat{\mathbf{n}} \mathrm{d} A=0
$$

where $\mathbf{Q}=[\rho, \rho u, \rho v, \rho w, \rho E]^{\mathrm{T}}$ is the vector of conserved state variables including the density $\rho$, the Cartesian momentum components, $\rho u, \rho v$ and $\rho w$, and the total energy per unit mass, $E ; \mathbf{F}$ is the inviscid flux tensor; and $\hat{\mathbf{n}}$ is the outward facing unit normal. The thermodynamic properties of air - namely pressure, sound speed and temperature - are obtained from curve fits of tabulated data from the work of Srinivasan ${ }^{36}$ since the perfect gas equation of state is not appropriate at meteoroid entry conditions. 
The Euler equations are solved with a finite-volume method on a Cartesian mesh with embedded boundaries $37[38$ The volume mesh consists of hexahedral cells, except for a layer of cut cells, which are cells clipped into arbitrarily shaped polyhedra by the meteoroid's surface, as illustrated in Fig. 2. The meteoroid geometry is specified by a watertight surface triangulation. The spatial discretization uses a cell-centered, secondorder accurate, finite-volume method with a weak imposition of boundary conditions. Steady-state solutions are obtained using a five-stage Runge-Kutta scheme accelerated by local time stepping, multigrid and parallel computing $39 \sqrt{40}$

To set the radius at which the signature is extracted, we follow the work of ReVelle 6 [7] and Henneton et al., 18 and use the blast radius of a cylindrical line source as a guide. The blast radius represents the distance from the body at which the shock overpressure ratio falls below unity, $\Delta p / p_{\infty}<1$, where $p_{\infty}$ is the freestream (undisturbed ambient) pressure. Strong-shock overpressures have $\Delta p / p_{\infty}>10$, so at a relaxation distance of one blast radius the pressure signature is well outside the region of strongly nonlinear shocks. When energy deposition is dominated by drag, a simple approximation of the blast radius is the product $M_{\infty} D$, where $M_{\infty}$ is the meteoroid's freestream Mach number and $D$ the diameter. While this condition is not rigorous, we find minimal variation in the propagated waveforms when the nearfield signatures are extracted at or beyond this distance. Moreover, for meteoroid sizes of a few centimeters the blast radius is on the order of a few meters to tens of meters. This is much smaller than the atmospheric scale height, which justifies the use of a uniform atmosphere in the nearfield.

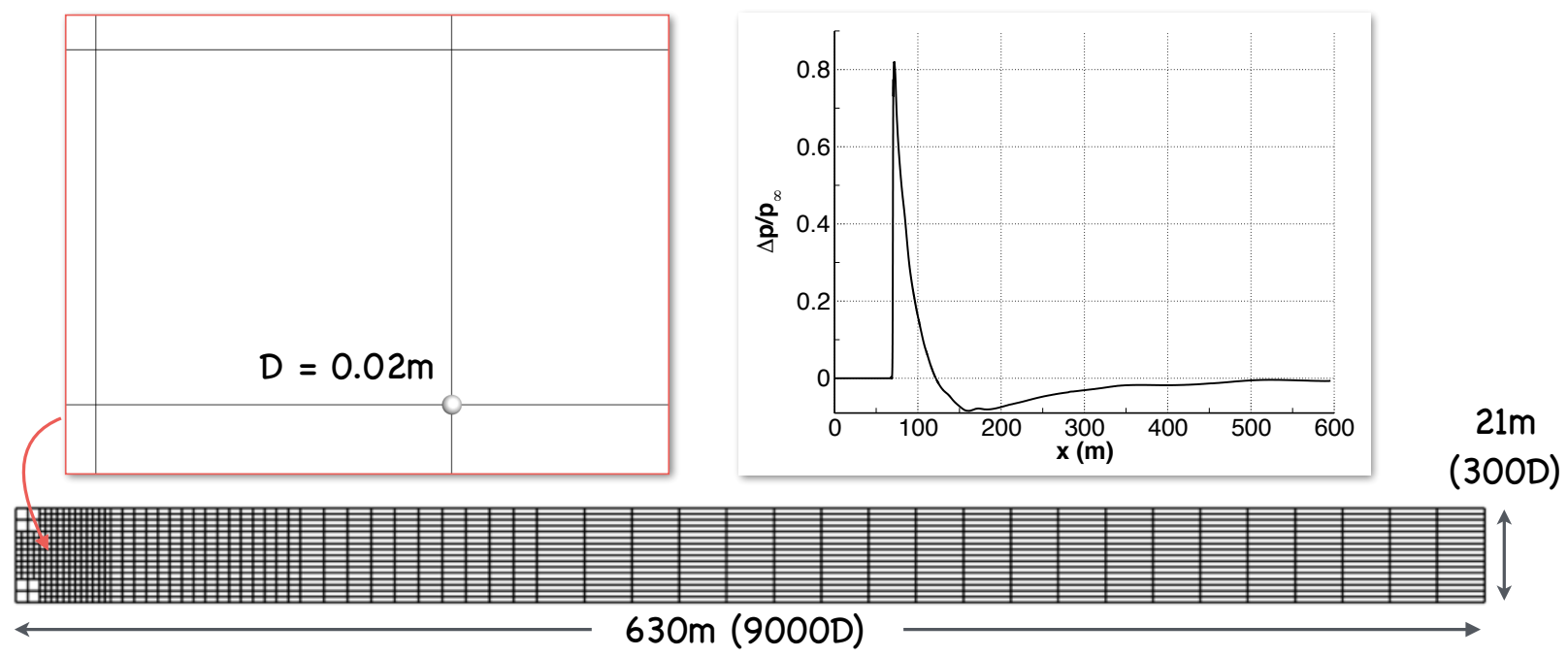

Figure 3. Background Cartesian mesh used for nearfield simulation. A representative nearfield signature is shown on top-right and a zoom of the nearbody region is on the top-left. Note the increasing cell aspect ratio in the streamwise direction in the bottom frame.

The primary challenge of the nearfield simulation is the highly anisotropic flowfield. To explain, consider a spherical meteoroid with diameter $D=2 \mathrm{~cm}$ at $M_{\infty}=60$. At these conditions, the blast radius is $1.2 \mathrm{~m}$, or 60 body-lengths, and the Mach angle is $\approx 1^{\circ}$. This means that the bow shock will reach the extraction location of the nearfield signature approximately $72 \mathrm{~m}$ downstream from the meteoroid, or 3,600 body-lengths. Figure 3 shows that the computational domain must continue well downstream of the leading shock to capture the over-expansion and recompression of the blast wave. A representative signature is shown at the top-right of Figure 3, where overpressure is plotted as a function of the streamwise position. The bottom frame of Figure 3 shows the computational domain together with the background (initial) Cartesian mesh used in this work. We set the domain length to minimize truncation of the aft pressure recovery region. Note the factor 
of 30 difference between the crossflow and streamwise directions. In addition, note that the streamwise cell aspect ratio is prescribed to increase from isotropic cells near the meteoroid to cells with an aspect ratio of 8 to take advantage of the alignment of the bow shock with the mesh (Mach angle $\approx 1^{\circ}$ ) as it propagates downstream. The top-left frame shows a close-up of the near-body region of the initial mesh highlighting the small size of the meteoroid relative to the scale of the domain.

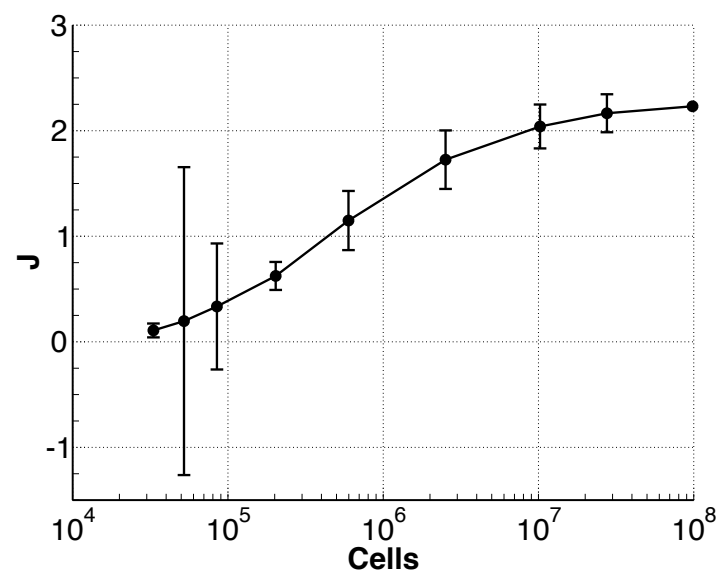

(a) Functional convergence for perfect gas simulation

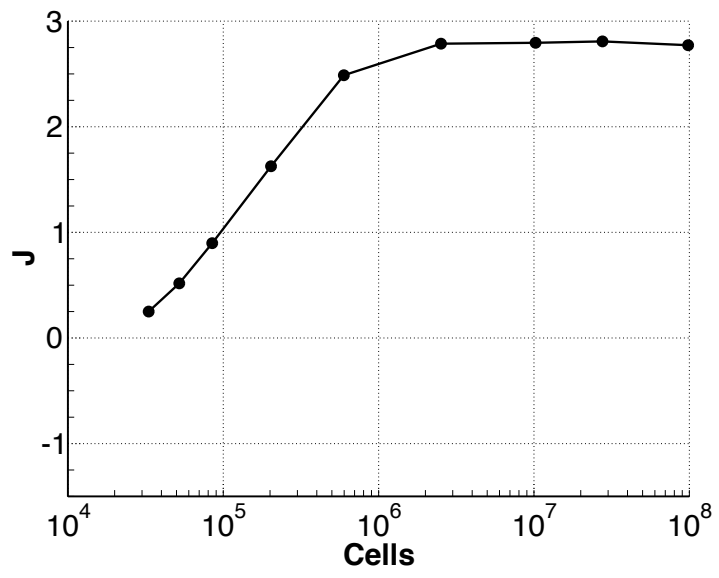

(b) Functional convergence for equilibrium gas simulation

Figure 4. Convergence of signatures driving the mesh adaptation procedure for perfect gas (left) and equilibrium gas (right) simulations. Error bars indicate level of discretization error in the perfect gas (left) simulation.

We address the wide range of scales of the nearfield domain through use of adaptive mesh refinement based on the method of adjoint-weighted residuals. $\frac{41}{1}$ The strength of this method is its ability to refine only those cells of the computational domain that most affect the level of discretization error in the nearfield signature. We use a linear combination of two line sensors to drive the mesh adaptation procedure: the primary sensor is one blast radius below the meteoroid while a secondary sensor is positioned at $3 / 4$ of a blast radius to improve mesh smoothness near the primary sensor. The functional associated with each line sensor is given by

$$
J=\int_{0}^{L}\left(\frac{p-p_{\infty}}{p_{\infty}}\right)^{2} d \ell
$$

Figure 4 shows convergence of the adaptation functional with mesh refinement for one of the meteor cases presented later (Meteor 20080325) as a representative example. Since the adjoint error estimation method is not implemented for equilibrium gas simulations, we use perfect gas simulations with a modified ratio of specific heats, $\gamma$, to build the mesh. Thereafter, we execute the equilibrium gas simulations on the same sequence of meshes and monitor convergence of the signature. Figure 4 shows that the signatures begin to convergence on a mesh with approximately 10 million cells. We perform two additional adaptation cycles and use meshes with approximately 100 million cells to evaluate the nearfield signature. Figure 5 shows an example of the final mesh in the nearbody region of a spherical meteoroid. These problems require approximately $24 \mathrm{~h}$ to mesh, adapt, and solve on a computer with 28 cores

\section{B. Farfield Domain}

The nearfield signature is propagated through the atmosphere via a two-step procedure. The first step involves ray tracing to compute the path of the signature from the meteoroid trajectory to the location of the microphone array. The second step involves determining the shape of the signature as it travels along this ray path. We use the sBOOM code by Rallabhand ${ }^{42}$ for both steps of the propagation.

Figure 6 shows an example ray path from the meteoroid to the observer. The path is generally curved due to the effects of atmospheric stratification and wind. The launch direction of the ray is normal to the meteoroid's cylindrical Mach-cone, consistent with our assumption of energy deposition being dominated by the drag of a single-body meteoroid The range for the off-track angle is constrained between $-90^{\circ}$

\footnotetext{
${ }^{\mathrm{b}}$ Intel Xeon E5-2680v4 (Broadwell) processors.

${ }^{\mathrm{c}}$ Actual observations 11 show that the launch angle can deviate by as much as $20^{\circ}$ from the normal.
} 


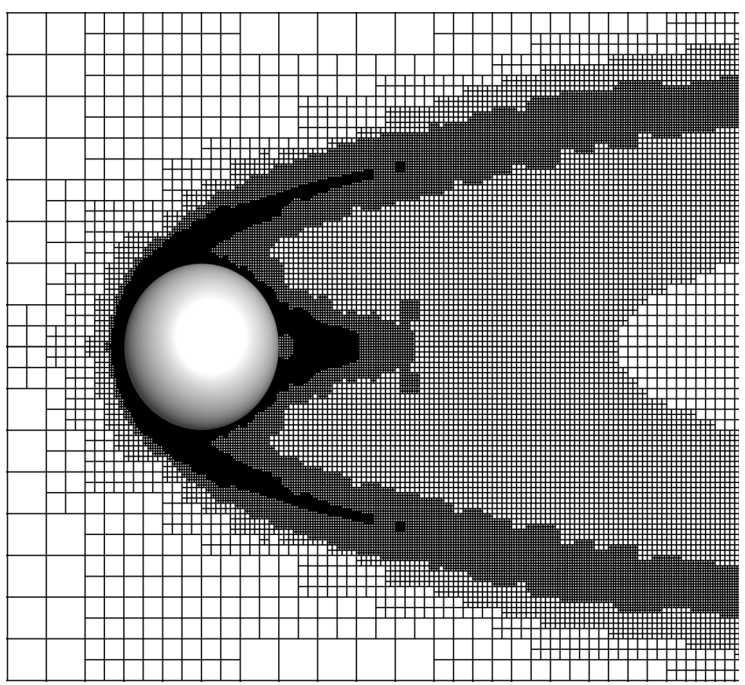

Figure 5. Final mesh in the near-body region of a spherical meteoroid $\left(M_{\infty}=42.5\right.$, equilibrium air model).

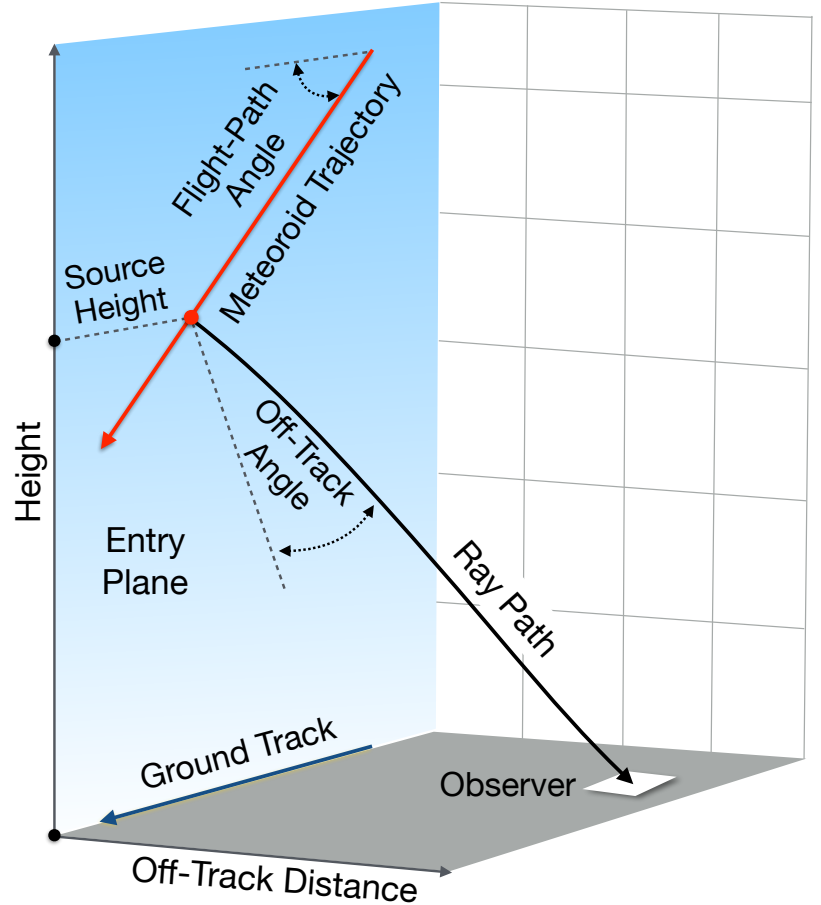

Figure 6. Acoustic ray path from a source point on the trajectory to the observer. 
and $90^{\circ}$, depending on the location of the observer. As indicated in Figure 6 this means that only "direct arrivals" are considered. These are signatures along ray paths that are initially cast in a downward direction from the trajectory. Secondary paths due to refraction in the upper atmosphere are neglected because these are generally much weaker. This limits the wave propagation distance to about $300 \mathrm{~km}$, thereby limiting atmospheric variabilities that arise in long-range propagation, e.g., at distances of 1,000s of kilometers.

For given flight path angle, observer location, atmospheric conditions, and measured travel time, we search for the unique source height with a direct ray path from the trajectory to the observer. The ray tracing proceeds by marching down the trajectory and finding an off-track angle for which the ray-path ground intercept is within some small radius of the observer (typically one kilometer). The travel-time residual between the computed and observed values is monitored and the source height is adjusted until the residual is minimized. This establishes the travel time of the signature to within a few seconds, i.e, within $1 \%$ for the events presented in this work.

If a ray path is not found, Silber et al ${ }^{[15}$ suggest that this is an indication that the observed signature does not originate from the cylindrical Mach-cone, but is instead from a more omnidirectional shock front, such as a quasi-spherical wave, that may arise during the disintegration of the meteoroid. To test this, Silber et al ${ }^{\sqrt{15}}$ perturb the ray launch direction away from the Mach-cone normal and look for viable ray paths. We use a similar procedure in Ref. 24 , which involves artificially perturbing the flight path angle to affect the ray launch direction. This is especially useful when dealing with observations of multiple signatures from the same trajectory. The ray-tracing procedure allows us to identify which signature is most likely from the cylindrical Mach-cone generated by the meteoroid's ballistic flight.

The evolution of the waveform as it descends to the ground is modeled through use of the augmented Burgers' equation. $\frac{42}{45}$ In dimensionless form, the equation is given by

$$
\frac{\partial p}{\partial \sigma}=p \frac{\partial p}{\partial \tau}+\frac{1}{\Gamma} \frac{\partial^{2} p}{\partial \tau^{2}}+\sum_{\nu} C_{\nu}\left(1+\theta_{\nu} \frac{\partial}{\partial \tau}\right)^{-1} \frac{\partial^{2} p}{\partial \tau^{2}}-\frac{p}{2 S} \frac{\partial S}{\partial \sigma}+\frac{p}{\rho_{0} c_{0}} \frac{\partial\left(\rho_{0} c_{0}\right)}{\partial \sigma}
$$

where $p$ is pressure, $\sigma$ the distance, $\tau$ the time, $C_{\nu}$ the dispersion parameter, $\Gamma$ the thermoviscous parameter, $\theta_{\nu}$ the molecular-relaxation time parameter of the $\nu^{\text {th }}$ relaxation process, $\rho_{0}$ and $c_{0}$ the local density and speed of sound, respectively, and $S$ the ray tube area of adjacent ray paths. The equation is solved in the time domain using operator splitting and a finite-difference discretization. ${ }^{42}$

By including classical and molecular-relaxation losses, in addition to the stratification, spreading and nonlinear terms, the solution of the augmented Burgers' equation directly predicts shock rise-timd This is important because the rise-time determines the frequency spectrum of the signature. Figure 7 illustrates the significant role of the molecular-relaxation terms in Eq. 3. Meteoric signatures are much weaker and originate from much higher altitudes than aircraft sonic-booms, and we find the attenuation due to molecular-relaxation to be much more pronounced. Figure 7 shows two meteoric ground signatures propagated from a height of $52 \mathrm{~km}$ with and without the relaxation terms in Eq. 3. These losses reduce the zero-peak amplitude by almost a third, approximately triple the shock rise-time and increase the time to the zero-crossing in the expansion by approximately $14 \%$. Moreover, Silber et al $\frac{15}{15}$ report large differences in

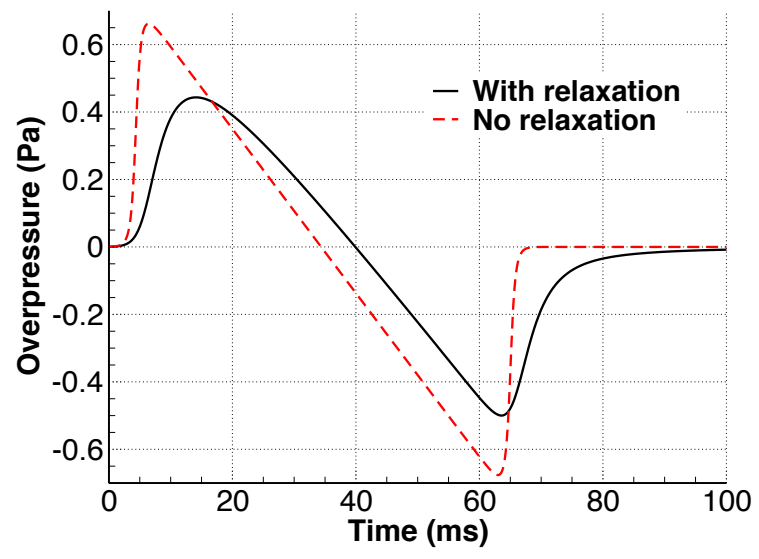

Figure 7. Ground signatures with and without molecular relaxation losses. maximum amplitude when comparing their observations to predictions based on an improved version of the ReVelle model ${ }^{7]}$ The general formulation of the augmented Burgers' equation provides a potentially more accurate approach. We note that the absorption coefficients of Sutherland and Bass $\$ 46$ are not used, but this should not be a significant source of error since the source heights for the selected cases are all below $80 \mathrm{~km}$.

sBOOM is routinely applied in aircraft sonic-boom prediction studies using established "best-practice" guidelines $\frac{33}{3}$ Typically, the time step for temporal discretization of Eq. 3 is set internally within sBOOM.

\footnotetext{
${ }^{\mathrm{d}}$ Shock rise-time is the time between the overpressure onset and the first peak in the signature.
} 


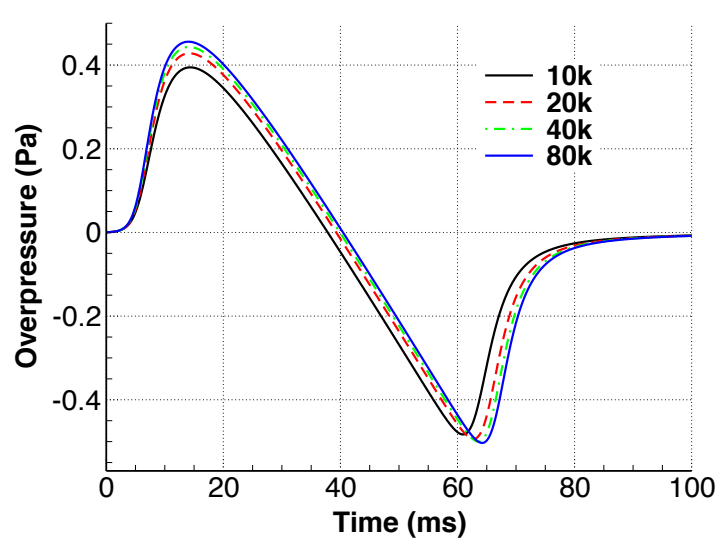

(a) Grid refinement study $(10,000-80,000$ points $)$

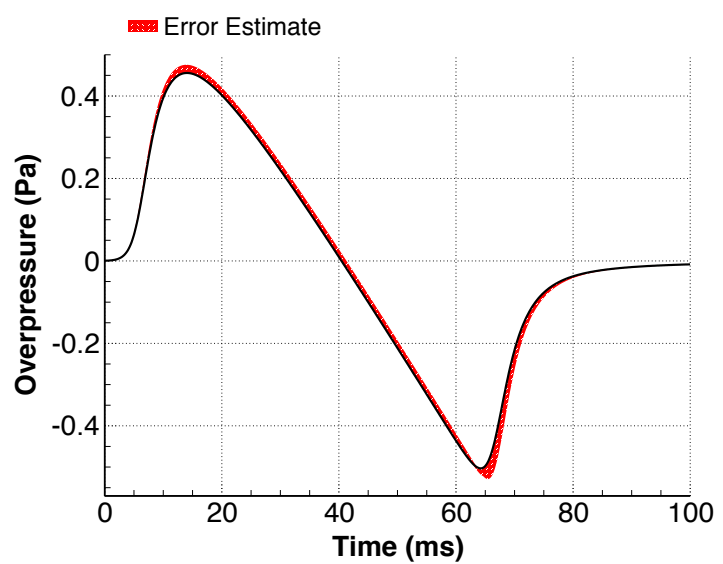

(b) Finest $(80,000)$ point signature with discretization error estimate

Figure 8. sBOOM grid refinement study (left) and an extrapolated discretization error estimate for signature on the finest-mesh (right). Propagation from $H=52 \mathbf{~ k m}$ and range of $\mathbf{7 4} \mathbf{~ k m}$.

Spatial discretization, however, is user-controlled. We performed several verification studies to determine the number of points needed to obtain grid-converged ground signatures. Figure 8 shows an example gridconvergence study for a nearfield signature similar to the one shown in the top-right frame of Figure 3 propagated from a height of $52 \mathrm{~km}(170,000 \mathrm{ft})$ and over a range of approximately $74 \mathrm{~km}$. We double the number of points in the signature starting from 10,000 points, labeled "10k" in Figure 8(a). Before propagating the signature, its aft portion is linearly ramped to the freestream pressure over a distance of approximately $400 \mathrm{~m}$ and then zero-padded to a distance of approximately $3 \mathrm{~km}$ to ensure that the signature remains in the computational domain when it reaches the ground. Figure $8(\mathrm{a})$ shows that the changes in the ground signature are very small once the discretization reaches 40,000 points. We use the last three signatures (20k-40k-80k point sets) to estimate the remaining discretization error in the finest signature by applying Richardson extrapolation $\underline{47[48}$ For these three signatures we calculate an observed order of accuracy just below unity, assuming that the spatial discretization error dominates the temporal error. Figure 8(b) shows that the remaining error is small. Based on this and additional grid refinement studies, we use 40,000 points to propagate all signatures presented in the Results section. This results in a $50 \mathrm{kHz}$ resolution of the signal at the ground.

\section{Method Validation - Stardust Entry}

We simulate the entry of the Stardust capsule to establish the accuracy of our computational approach on a highly constrained case. As part of its mission to collect dust particles, Stardust closely matched the orbit of comet $81 \mathrm{P} /$ Wild-2. Consequently, its atmospheric entry mimicked that of a cometary meteoroid, and with a speed of $12.8 \mathrm{~km} / \mathrm{s}$, it remains one of the fastest entries of a man-made object. Crucially, unlike meteoroids, the capsule shape is known precisely, see for example Stackpoole et al $\stackrel{49}{4}$ Moreover, the capsule's

ballistic trajectory, pressure signature, and the atmospheric conditions are well documented, $22[23|50| 51$ and the energy deposition is dominated by aerodynamic drag since energy loss due to radiation is negligible at these conditions.

We test our computational approach by trying to reproduce the pressure measurements of Plotkin et al., 22 who used B\&K 4193 low-frequency microphones digitized at $24 \mathrm{kHz}$ to record a signature very close to the capsule's ground track. The entry occurred on 15 January 2006. The capsule shape was a $60^{\circ}$ half-angle spherically-blunted cone with a diameter $D=0.81 \mathrm{~m}$. The capsule reached the signature's source height, $H$, of $50.4 \mathrm{~km}$ at 09:57:49.078 UTC, by which point the capsule had decelerated to $6.4 \mathrm{~km} / \mathrm{s}$. This corresponds to a freestream Mach number of 19.4. The capsule's flight-path angle was constant at $8.2^{\circ}$ and its deceleration at the source height was $0.18 \mathrm{~km} / \mathrm{s}^{2}$.

Figure 9 shows the computed pressure field near the capsule. The flow is dominated by a detached bow shock, a thin shock layer, and weaker wake shocks that merge downstream. The blast radius of the capsule 


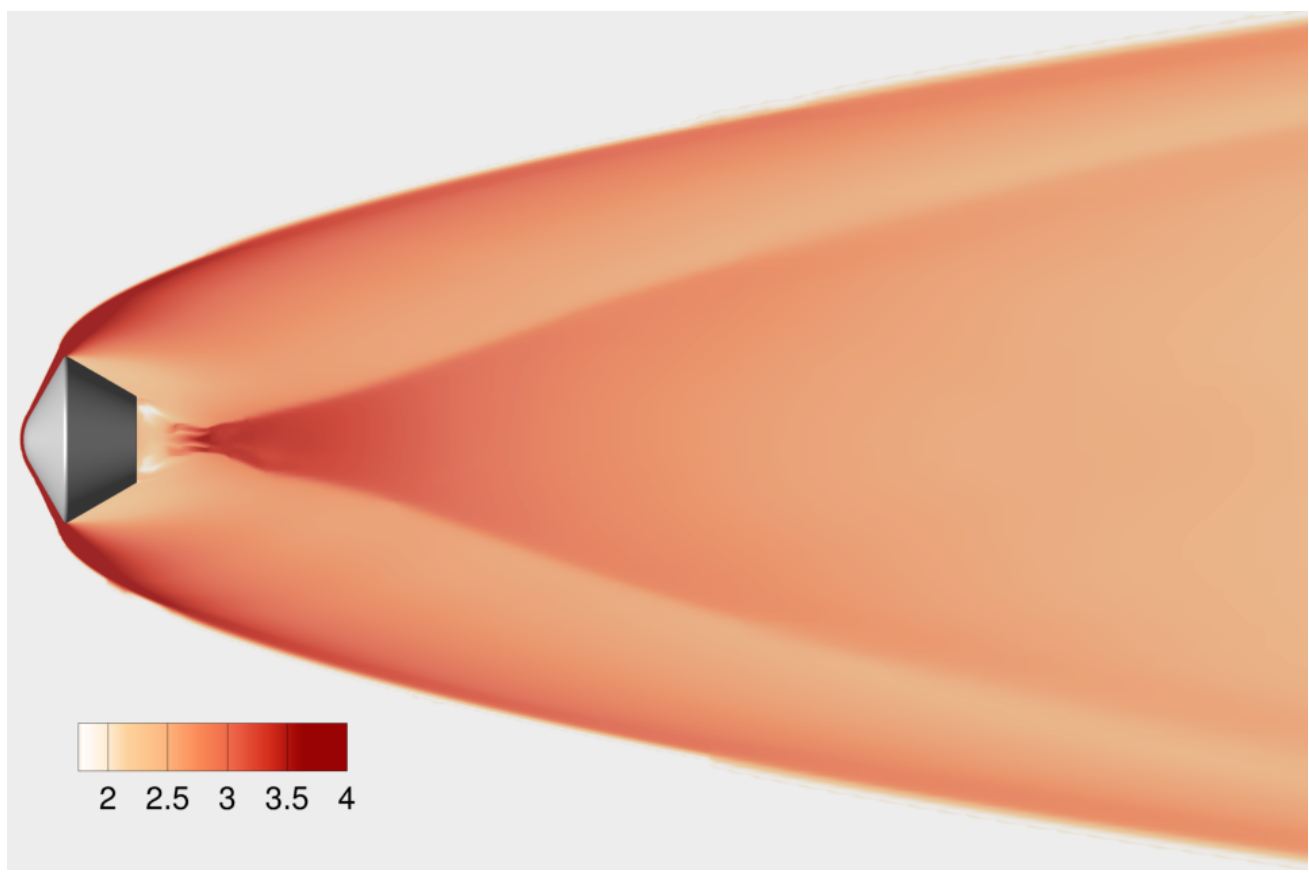

Figure 9. Pressure contours $\left(\log _{10} p\right)$ around the Stardust capsule $\left(M_{\infty}=19.4, H=50.4 \mathbf{k m}, p_{\infty}=72.2 \mathrm{~Pa}\right.$, $D=0.81 \mathbf{m}$, equilibrium air model).

is $15.7 \mathrm{~m}$, or $19.4 D$, at the source height. Figure 10 shows the nearfield signature extracted streamwise at a distance of $20 \mathrm{D}$ below the capsule, or slightly over one blast radius away. The time (in milliseconds) on the $x$-axis is obtained by normalizing the distance along the sensor by the speed of the capsule. Observe that the shock overpressure ratio is well less than 1 , which indicates that the main shock is approaching the weakly nonlinear regime. Moreover, while the amplitude of the signature is dominated by the shock, its duration is dominated by the over-expansion and the slow recompression.

To propagate the nearfield signature to the ground, we use the recorded temperature and wind profiles from Desai and Qualls. ${ }^{50}$ Ray tracing reveals that due to the unusually strong winds that night, the ray launch direction is at an off-track angle of $4^{\circ}$ even though the microphone array is located essentially on-track. Figure 11 shows the attenuation and lengthening of the signature as it propagates through the atmosphere. The signature labeled "Nearfield" is the waveform after just $100 \mathrm{~m}$ of propagation, which is very similar to the one shown in Figure 10, except here we plot overpressure instead of overpressure ratio.

After about $1 \mathrm{~km}$ of propagation (red signature at height of $49 \mathrm{~km}$ in Figure 11), the peak has attenuated significantly and there is already a small increase in the duration of the positive pressure phase. After $10 \mathrm{~km}$ of propagation (green signature at height of $40 \mathrm{~km}$ ), the signature is a classic N-wave and the time to the zerocrossing has more than doubled. Counterintuitively, the amplitude thereafter increases with propagation distance.

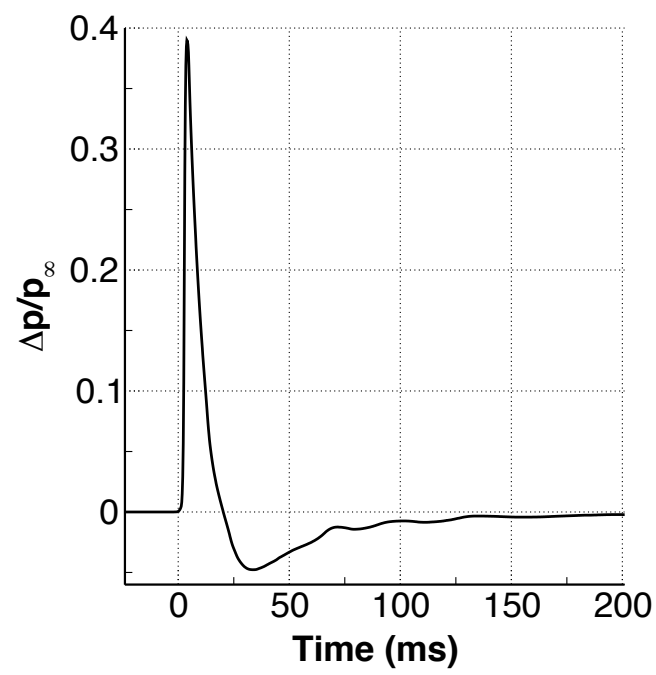
This is simply due to the exponentially increasing ambient pressure as the signature descends and should not be confused with the overpressure ratio, which always decreases. Over the last $20 \mathrm{~km}$ of propagation, there are essentially no changes in the duration of the positive pressure phase or the overall period. This indicates that the wave has reached the linear regime. 


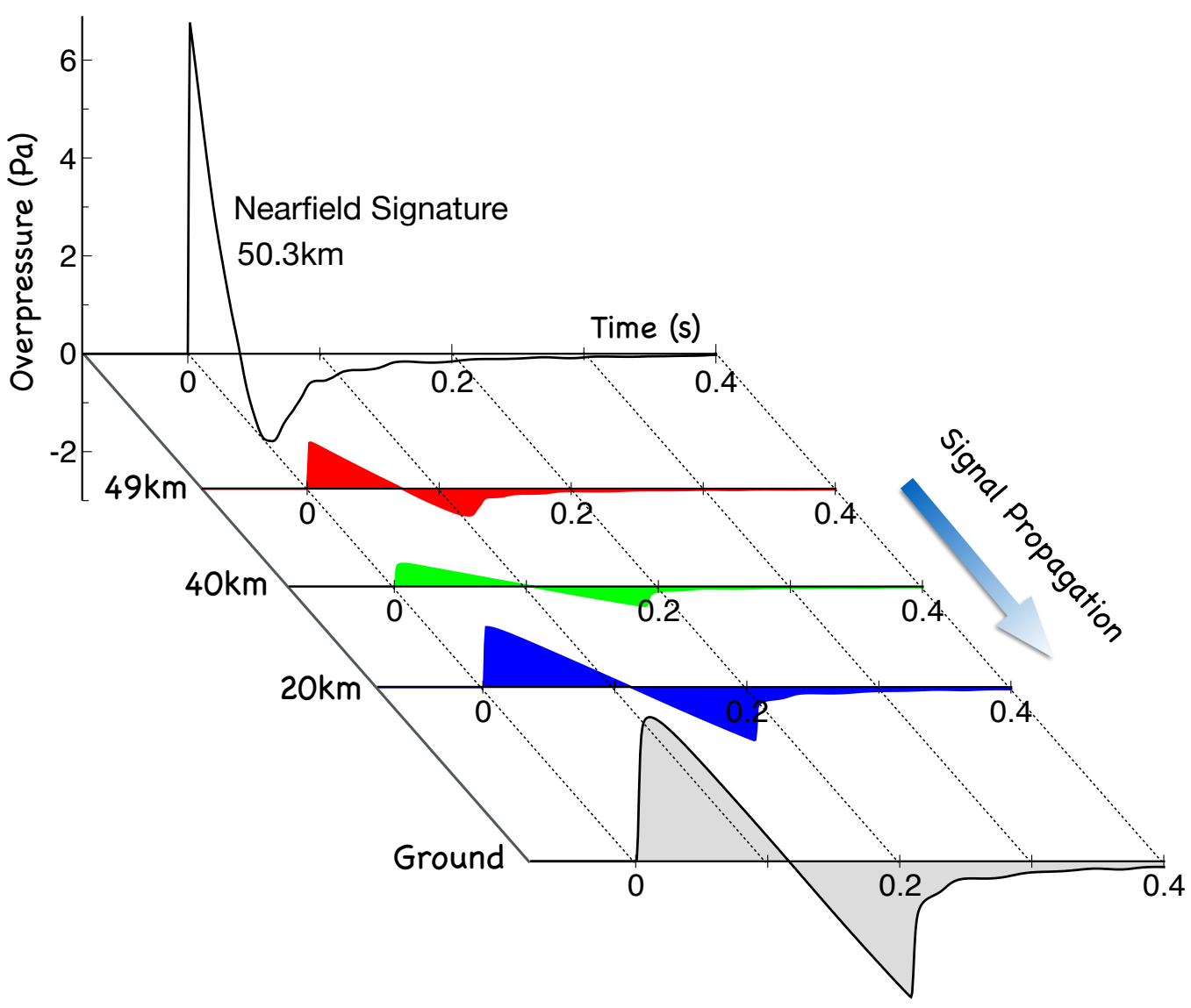

Figure 11. Evolution of the Stardust signature as it propagates to the ground through a stratified, windy atmosphere (constant ground reflection factor).

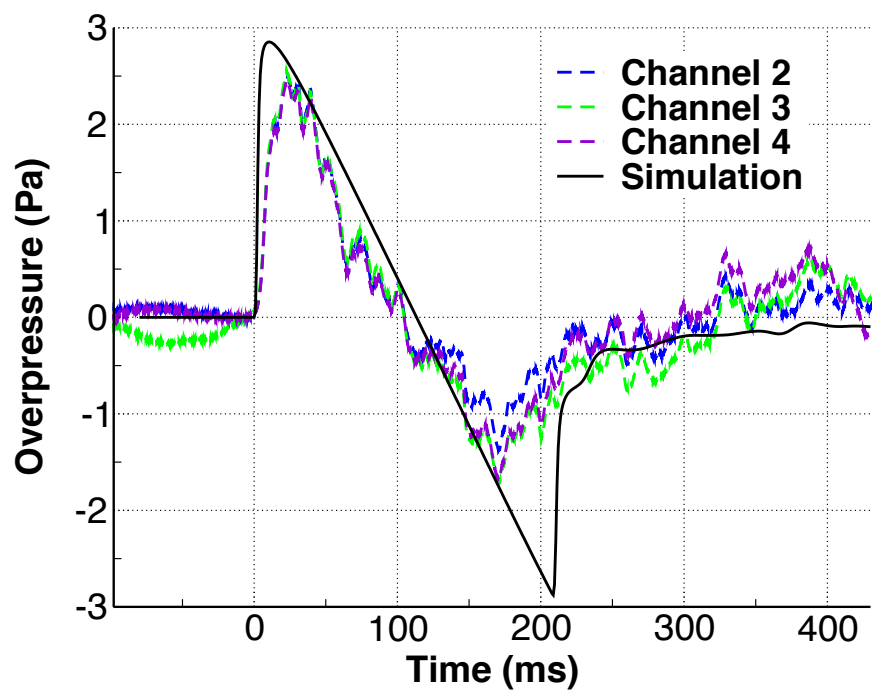

Figure 12. Observed and computed ground pressure signatures for the Stardust capsule. Each "Channel" corresponds to a sensing microphone. 
Figure 12 compares the computed ground signature with the measurements of Plotkin et al ${ }^{[22}$ Data from three microphones is shown, labelled "Channel 2" through "4". Overall, the agreement is excellent, especially in terms of the shock rise time and the duration of positive pressure phase. The maximum amplitude is overpredicted by about $10 \%$. This can be attributed to the rapidly changing atmospheric conditions due to a blizzard in the region of the microphone array, as well as to using the as-built capsule geometry in the simulation instead of the ablated shape $\mathrm{e}^{\mathrm{e}}$ The largest differences occur during the negative pressure phase. The expansion in the measured signature is not as deep, and the recompression to ambient pressure is much more gradual. These differences may be partially attributed to the modeling fidelity of the nearfield wake region in addition to the uncertainty in the atmospheric conditions. Nevertheless, there is good agreement in the signal period. Lastly, Plotkin et al ${ }^{[22}$ report a travel time of 161 seconds for the signature to reach the microphones and we compute a travel time of 162.2 seconds. The agreement is excellent; the computation is within $1 \%$ of the observed value, which is well within the uncertainty of the atmospheric model.

\section{Meteor Events}

We present three meteor events from the Silber et al ${ }^{15}$ dataset. Our primary selection criterion is a limit on the source height - we consider only events for which the source height does not significantly exceed 70 $\mathrm{km}$. For centimeter-sized meteoroids, this is on the boundary between the continuum and the transitional regime $(\mathrm{Kn} \approx 0.1)$, since the mean free path at $70 \mathrm{~km}$ is approximately $1 \mathrm{~mm}$. We also check each meteor's light curve and pick events for which the source height does not coincide with major peaks in the light curvef These correspond to flares that are usually associated with meteoroid fragmentation. Thorough analysis of pressure signatures from such events is beyond the scope of this work.

Table 1. A summary of entry parameters for the selected meteor events from Silber et al.15

\begin{tabular}{ccccccc}
\hline \hline Date & $\begin{array}{c}\text { Entry } \\
\text { Speed }^{\mathrm{a}} \\
\mathrm{km} / \mathrm{s}\end{array}$ & $\begin{array}{c}\text { Begin } \\
\text { Height } \\
\mathrm{km}\end{array}$ & $\begin{array}{c}\text { End } \\
\text { Height } \\
\mathrm{km}\end{array}$ & $\begin{array}{c}\text { Flight } \\
\text { Path Angle }^{\mathrm{b}} \\
\mathrm{deg.}\end{array}$ & $\begin{array}{c}\text { Entry } \\
\text { Mass }^{\mathrm{c}}\end{array}$ & $\begin{array}{c}\text { Entry } \\
\text { Diameter }^{\mathrm{d}} \\
\mathrm{cm}\end{array}$ \\
\hline 20080325 & 13.5 & 76.2 & 32.8 & $44.8^{\circ}$ & 917 & 7.8 \\
20081028 & 15.8 & 81.2 & 41.1 & $33.1^{\circ}$ & 110 & 4.7 \\
20090428 & 21.2 & 83.5 & 38.0 & $57.2^{\circ}$ & 330 & 6.8 \\
\hline \hline
\end{tabular}

${ }^{\text {a }}$ Meteoroid speed at the onset of ablation.

${ }^{\mathrm{b}}$ Measured from horizontal in the plane of entry, see Figure 6

${ }^{\mathrm{c}}$ Mass based on the FM model of Ceplecha and ReVelle. ${ }^{[27}$

${ }^{\mathrm{d}}$ See Table 3 for density estimates.

Table 1 summarizes the entry parameters of the selected meteors. Note that a flight path angle of $90^{\circ}$ represents a vertical entry and that the flight path angle is assumed to be constant along the trajectory. Table 2 summarizes the source heights of the signatures based on raytracing in Ref. 24, as well as the speed, deceleration, and remaining mass of each meteoroid at the source heights. These are primarily from Silber et al., ${ }^{15}$ who used the fragmentation model of Ceplecha and ReVelle ${ }^{27}$ to fit the observed light curves. The deceleration in all cases is relatively small, so the speed of the meteoroid at the source height is usually within $10 \%$ of its entry speed. The ablation effects are more significant, with meteors 20081028 and 20090428 losing almost $30 \%$ of their entry mass by the time they reach the source height. Note that Meteor 20090428 features two arrivals. The column labeled "Total Range" is the distance between the ELFO infrasound array and the source point on the trajectory. Hence, ELFO is 70 to $150 \mathrm{~km}$ away from the source points and the signatures take 4-8 min to travel there. Overall, the selected cases provide a range of signatures typical of many events.

To approximate the physical size of the meteoroid, we assume a spherical shape and use the bulk density estimates from Ceplecha et al. ${ }^{26}$ Table 3 summarizes the diameter of the meteoroid on entry and at the

\footnotetext{
${ }^{\mathrm{e}}$ We note that the recession depth was only a few millimeters, so the shape change due to ablation should not significantly affect the signature 49

${ }^{\mathrm{f}}$ Light curves for all events are available in Ref. 52 .
} 
Table 2. A summary of signature source heights, travel times, and trajectory parameters from Silber et al $\sqrt{15}$ and Nemec et al $\$ 24$

\begin{tabular}{ccccccc}
\hline \hline Date & $\begin{array}{c}\text { Source } \\
\text { Height } \\
\mathrm{km}\end{array}$ & $\begin{array}{c}\text { Total } \\
\text { Range }^{\mathrm{a}} \\
\mathrm{km}\end{array}$ & $\begin{array}{c}\text { Travel } \\
\text { Time }\end{array}$ & $\begin{array}{c}\text { Speed } \\
\mathrm{km} / \mathrm{s}\end{array}$ & $\begin{array}{c}\text { Deceleration } \\
\mathrm{km} / \mathrm{s}^{2}\end{array}$ & $\begin{array}{c}\text { Mass at } \\
\text { Source Height } \\
\mathrm{g}\end{array}$ \\
\hline 20080325 & 61 & 113.1 & 341 & 13.3 & 0.24 & 882 \\
20081028 & 53.7 & 73.3 & 240 & 15.0 & 1.6 & 77.6 \\
20090428 & 58.7 & 138.6 & 456 & 21.1 & 0.32 & 237 \\
20090428 & 70.9 & 140.6 & 460 & 21.2 & 0.05 & 330 \\
\hline \hline
\end{tabular}

${ }^{a}$ Distance from observatory to the source-height point on the trajectory.

source height. The reduction in the diameter for meteor 20080325 is negligible. For meteors 20081028 and 20090428, we simulate both sizes to approximately bound the effects of ablation on the ground signature.

Table 3. Meteoroid densities and diameters.

\begin{tabular}{cccc}
\hline \hline Date & $\begin{array}{c}\text { Bulk } \\
\text { Density }^{\mathrm{a}} \\
\mathrm{g} / \mathrm{cm}^{3}\end{array}$ & $\begin{array}{c}\text { Entry } \\
\text { Diameter } \\
\mathrm{cm}\end{array}$ & $\begin{array}{c}\text { Ablated } \\
\text { Diameter } \\
\mathrm{cm}\end{array}$ \\
\hline 20080325 & 3.7 & 7.8 & 7.7 \\
20081028 & 2 & 4.7 & 4.2 \\
20090428 & 2 & 6.8 & 6.1 \\
\hline \hline
\end{tabular}

${ }^{\text {a }}$ Estimated density based on the PE scale of Ceplecha et al ${ }^{26}$ for small camera data and further adjusted based on orbital parameters.

\section{Results}

All nearfield simulations are computed in three dimensions, but we take advantage of two-fold symmetry due to the assumed spherical shape. The nearfield signatures are extracted at a distance of $40 \mathrm{D}$. Note that the data from the four ELFO sensors are digitized at a frequency of $100 \mathrm{~Hz}$. To compare the measured signatures with the simulations, the measured signatures are filtered with a second-order Butterworth $1 \mathrm{~Hz}$ high-pass filter and are then phase aligned. For some of the events, not all sensors were functioning correctly and signatures from those sensors are not shown. The atmospheric conditions, namely temperature and wind, are identical to those used by Silber et al $\frac{15}{15}$ The mean atmospheric conditions for each event are defined by a spline fit between the United Kingdom Meteorological Office (UKMO) wind mode ${ }^{53}$ below $60 \mathrm{~km}$ and the HWM9554 and NRL-MSIS00 model 55 at high altitude. For atmospheric humidity, we use the model from ANSI S1.26, Annex C $\stackrel{56}{5}$ in all computations.

\section{A. $\quad$ Meteor 20081028}

We begin with Meteor 20081028. At the source height $H=53.7 \mathrm{~km}$, the freestream Mach number of the meteoroid is 45.6. Figure 13 shows normalized density contours near the body. Similar to the Stardust validation case (Figure 9), the flow is dominated by the bow shock. The shock stand-off distance is minuscule - the shock is within $2 \mathrm{~mm}$ of the body near the stagnation point and the post-shock density ratio exceeds the perfect gas limit by more than a factor of two.

Figure 14(a) shows the nearfield signatures extracted at a distance just under one blast radius away for the initial and ablated shapes. The overpressure ratios are less than 1, indicating that the signatures are outside the strong-shock regime. The signatures are very similar, with the signature from the smaller ablated 


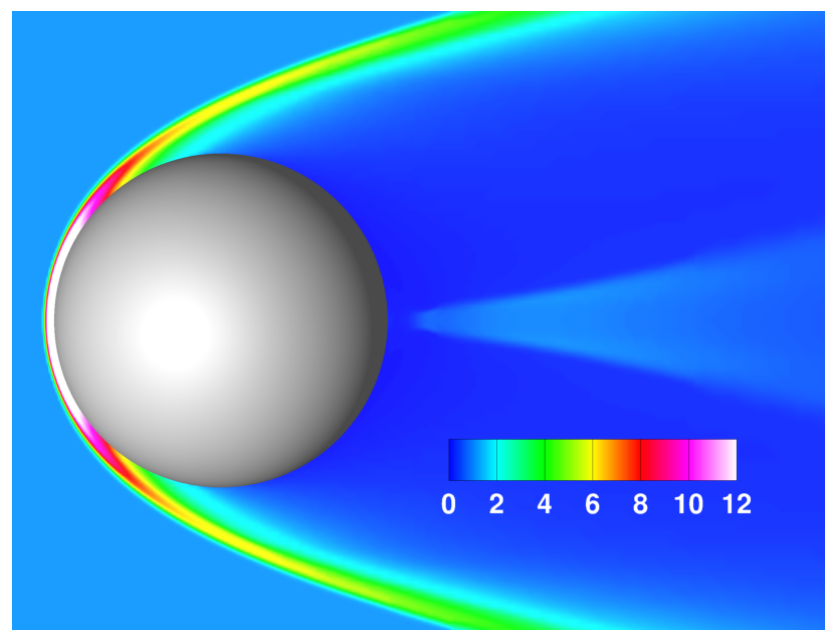

Figure 13. Contours of normalized density, $\rho / \rho_{\infty}$, on symmetry plane for Meteor $20081028(D=4.2 \mathrm{~cm}$, $\left.M_{\infty}=45.6, H=53.7 \mathrm{~km}\right)$.

shape generating a slightly lower peak and having a slightly shorter duration (earlier zero-crossing) of the positive pressure phase.

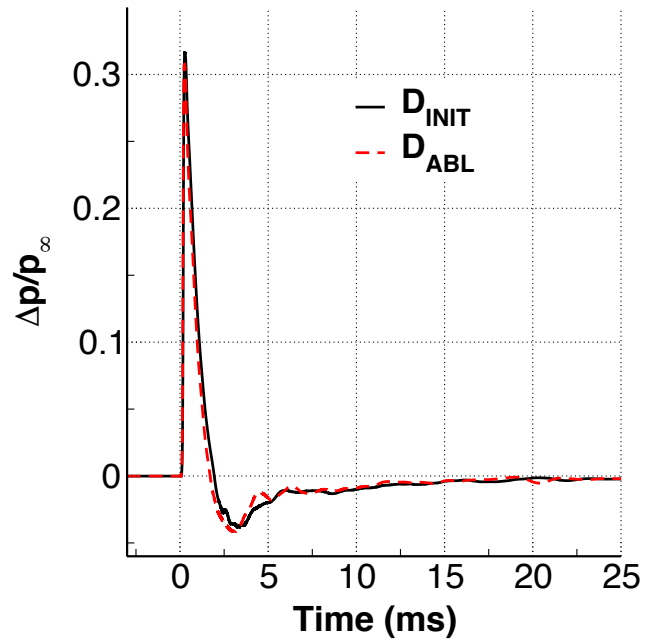

(a) Nearfield pressure signature

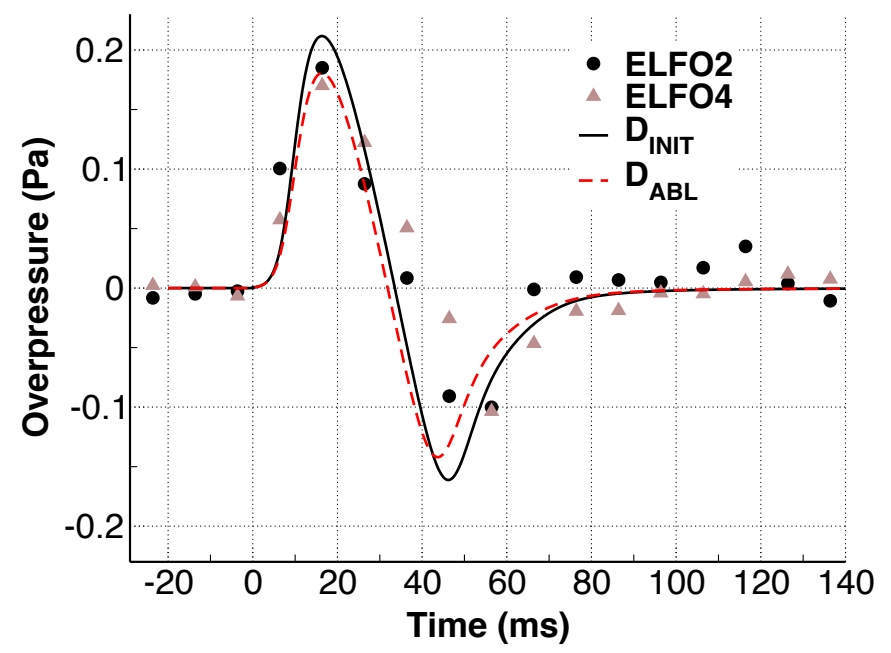

(b) Ground pressure signature

Figure 14. Nearfield and ground pressure signatures for Meteor 20081028. Results for the initial and ablated shapes are shown, labeled "DINIT" and "D $\mathrm{ABL}$ ", as well as observations from two ELFO sensors, labeled "ELFO2" and "ELFO4". Nearfield signature extracted at distance of $40 D\left(M_{\infty}=45.6\right.$ and $\left.H=53.7 \mathrm{~km}\right)$.

Figure 14(b) shows the computed and two observed ground signatures. The agreement is excellent in terms of the shock rise-time, the zero-peak amplitude, the pressure recovery, and the period. The duration of the positive pressure phase is underpredicted by approximately $10 \%$. The pressure signature of the ablated shape essentially matches the peak amplitude of the observed waveform. The largest deviations are in the slope and depth of the expansion. This is likely caused by uncertainties in the shape of the meteoroid and the atmospheric conditions, as well as the fidelity of the simulation in the wake region of the meteoroid.

The ground signature is a classical N-wave, quite similar to the Stardust signature shown in Figure 12 except much weaker. This is primarily due to the diameter of the meteoroid being roughly 20 times smaller than that of the capsule. The rounding of the shock discontinuities is much more pronounced in the meteoroid's signature. This stems from the significantly longer propagation distance and the greater height of the source point, which allows the molecular relaxation process to dominate the nonlinear steepening of the signature. 
B. Meteor 20090428

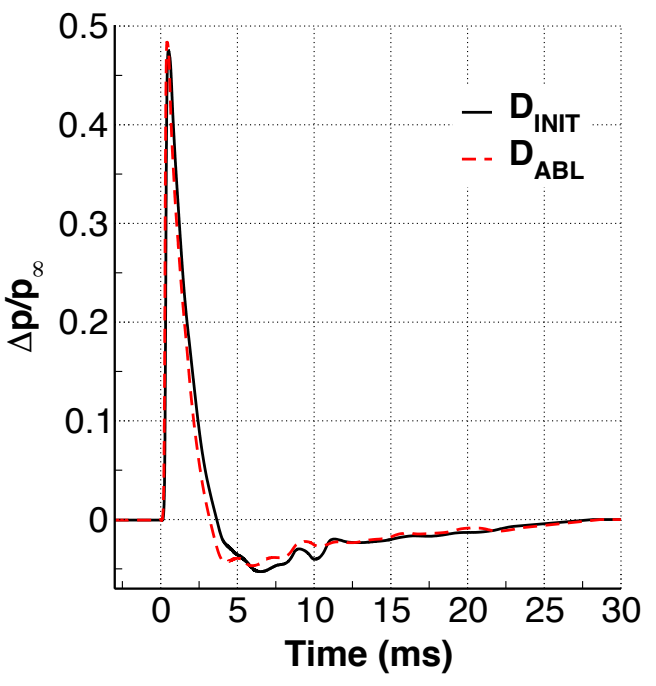

(a) Nearfield pressure signature

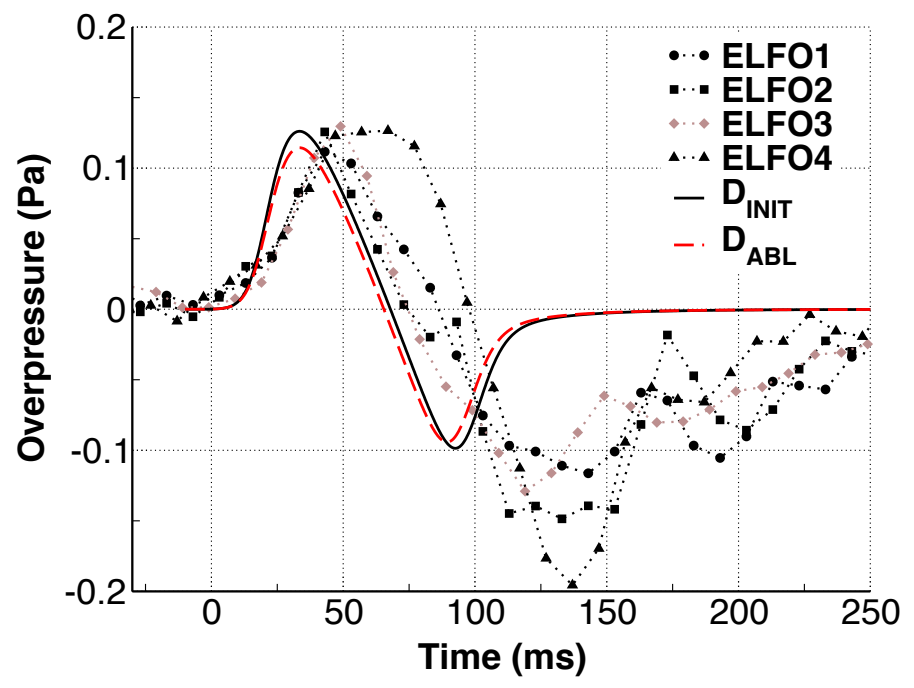

(b) Ground pressure signature

Figure 15. Nearfield and ground pressure signatures for the first arrival from Meteor 20090428. Results for the initial and ablated shapes are shown, labeled "D $\mathrm{D}_{\mathrm{INIT}}$ " and "D $\mathrm{D}_{\mathrm{ABL}}$ ", as well as ELFO observations from 4 sensors. Nearfield signature extracted at distance of $40 D\left(M_{\infty}=67.5\right.$ and $\left.H=58.7 \mathrm{~km}\right)$.

Recall that two arrivals were detected for Meteor 20090428, as summarized in Table 2. We start with the first arrival, which is from the cylindrical Mach-cone associated with the ballistic flight of the meteor.24 The source height is $58.7 \mathrm{~km}$ and the freestream Mach number of the meteoroid is 67.5. We simulate both the initial, $D=6.8 \mathrm{~cm}$, and ablated, $D=6.1 \mathrm{~cm}$, shapes. Figure $15(\mathrm{a})$ shows the nearfield signatures for both the initial and ablated shapes. The overpressure ratios are small and indicate that the shocks are approaching the weakly-nonlinear regime. As expected, the duration of the positive pressure phase of the ablated shape is slightly shorter than that of the initial shape. The small fluctuations visible in the pressure recovery region are from the wake of the meteoroid. They are primarily modeling and numerical artifacts that quickly dissipate as the signature propagates through the atmosphere.

Figure 15(b) shows the computed signature compared to the ELFO measurements. There is excellent agreement in the amplitude of the positive pressure phase. The shock rise time is underpredicted - the main discrepancy is at the foot of the shock where the measurements show an unusually slow ramp up. The measurements also show a wide spread of approximately $30 \mathrm{~ms}$ in the duration of the positive pressure phase and there is a factor of two variation in the depth of the expansion. Nevertheless, the computation is within $8 \%$ of the observations for two of the four ELFO sensors in terms of positive phase duration and peak-to-peak amplitude. The main difference between the computed and observed signatures is the slope of the trailing recompression. The observed signature takes much longer to recover and contains significant oscillations. This discrepancy most likely stems from our assumption of a single spherical body in the nearfield. This limits the ground signature to a more symmetric waveform than that of the measured signature.

Figure 16 shows the nearfield and the ground pressure signature of the later arrival from the higher source height $H=70.9 \mathrm{~km}$. The signature for only the initial shape is examined because this is sufficiently early in the trajectory where ablation is negligible. The meteoroid's freestream Mach number is 72.1. The overpressure ratio in Figure 16(a) is slightly higher than that of the first arrival shown in Figure 15(a) due to the higher Mach number.

Note that this arrival is not purely from the cylindrical Mach-cone of the meteoroid (see ray tracing results in Ref. 24). This is also supported by the light curve of this event, see Silber, ${ }^{52}$ which shows an inflection point near this source height that is consistent with high altitude quasi-continuous fragmentation. Despite these circumstances, Figure 16(b) shows that there is reasonable agreement between the computed and observed signatures. In particular, the duration of the positive pressure phase and the shock rise time agree well. The amplitude is underpredicted by approximately a factor of two. This is reasonable since the simulation does not model energy deposition due to fragmentation. In addition, this analysis helps explain 


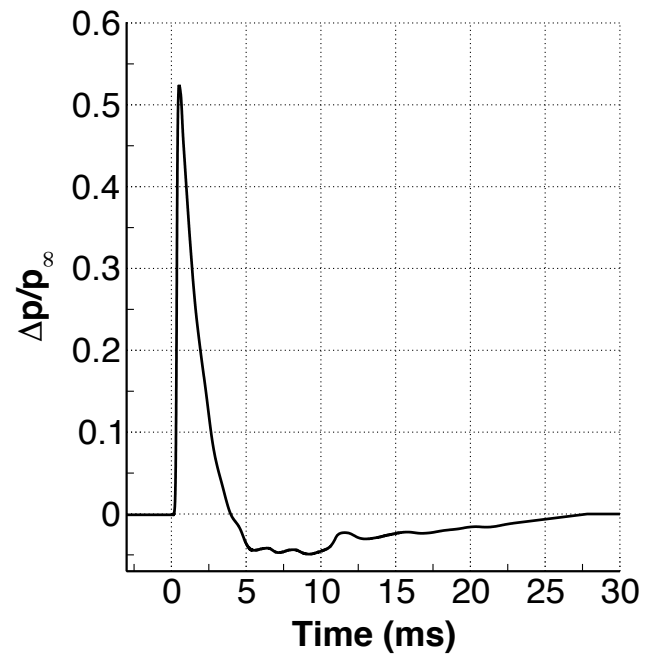

(a) Nearfield pressure signature

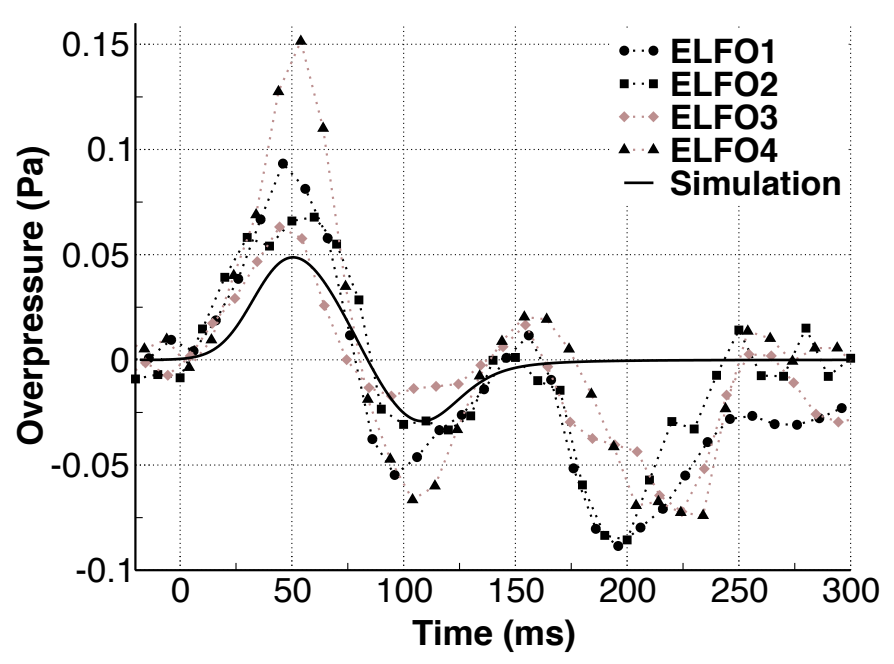

(b) Ground pressure signature

Figure 16. Nearfield and ground pressure signatures for the second arrival (source height $70.9 \mathrm{~km}$ ) from Meteor 20090428. Results for the initial (nonablated) shape and ELFO measurements are shown. Nearfield signature extracted at distance of $40 D\left(M_{\infty}=72.1\right.$ and $\left.H=70.9 \mathrm{~km}\right)$.

some of the discrepancies noted in the first arrival, since the partial disintegration of the meteoroid at the higher altitude means that the assumption of a spherical shape is most likely inappropriate at the lower altitude.

C. Meteor 20080325

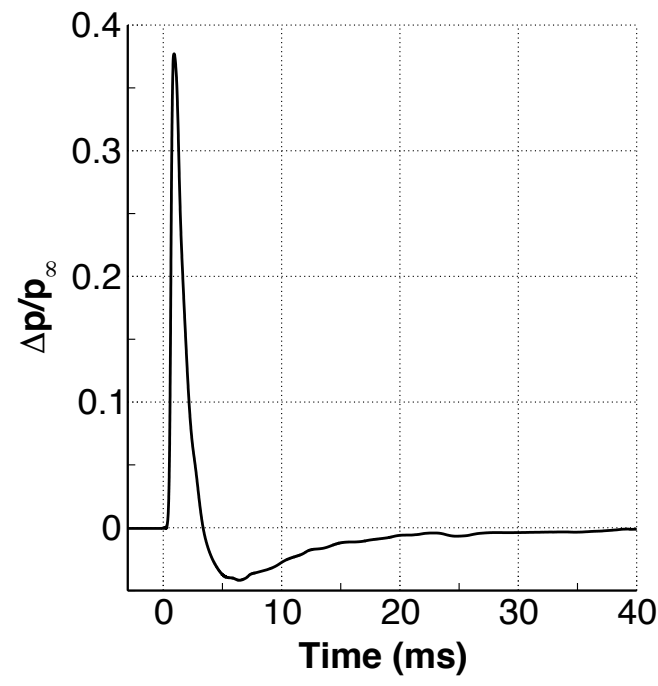

(a) Nearfield pressure signature

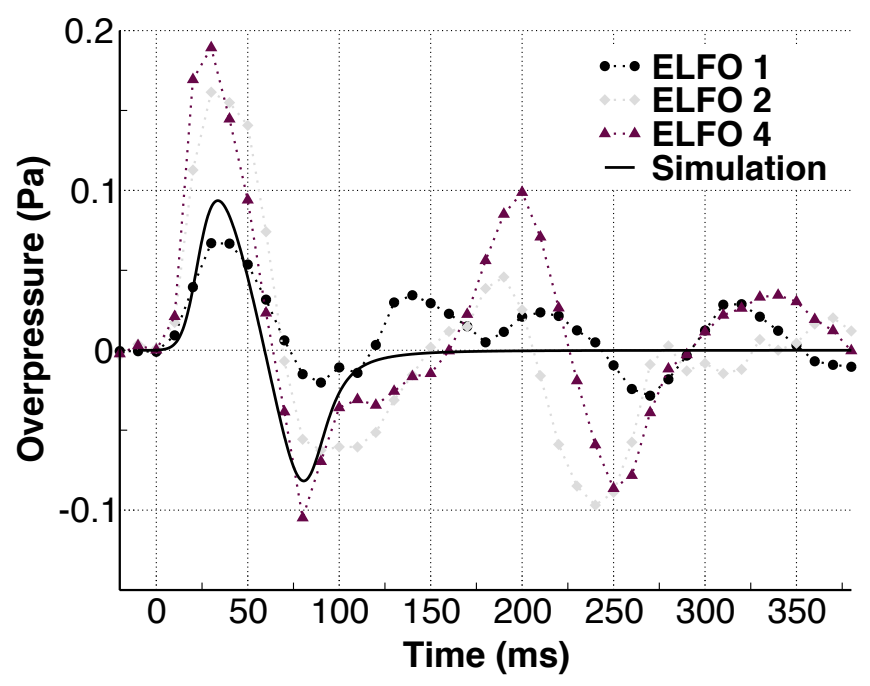

(b) Ground pressure signature

Figure 17. Nearfield and ground pressure signatures with ELFO measurements for Meteor 20080325. Nearfield signature extracted at distance of $40 D\left(M_{\infty}=42.5\right.$ and $\left.H=61 \mathrm{~km}\right)$.

Meteor 20080325 is the largest $(D \approx 8 \mathrm{~cm})$ meteoroid of this study. At the source height of $61 \mathrm{~km}$, the freestream Mach number is 42.5. The ray tracing for this event in Ref. 24 revealed that the source of the signature is a combination of the Mach cone plus some ablation or fragmentation effects. Figure 17 shows the nearfield and the ground pressure signature. The nearfield signature is quite similar to that of Meteor 20081028 shown in Figure 14(a). Figure 17(b) shows a significant spread in amplitude measurements among 
the three sensors. The computed zero-peak amplitude matches one of the sensors well, but underpredicts the other two sensors by almost a factor of 2 . The shock rise time is predicted accurately, which suggests that the source-height estimate is correct based on the level of attenuation of the leading shock. The duration of the positive pressure phase is only slightly underpredicted. The simulation, however, misses the trailing part of the waveform, where the measurements show additional compressions and expansions. We attribute this to the simple spherical shape assumed in the nearfield simulation.

\section{Conclusions}

We have validated a computational approach for predicting meteoric pressure signatures. Overall, the simulations perform remarkably well, considering that the observed signatures are generated by centimetersized bodies, and originate from altitudes of at least 50 kilometers and at ranges of about 100 kilometers from the observatory. The approach involves numerically solving the steady, three-dimensional Euler equations to initialize the propagation of a pressure signature through a stratified and windy atmosphere to the ground by using the augmented Burgers' equation. The use of mesh adaptation in the nearfield domain enabled sharp resolution of the pressure signature at large off-body distances, which significantly reduced simulation cost and improved accuracy. The farfield solver required a relatively fine discretization between 40,000 and 80,000 points (corresponding to ground signal resolution of $50-100 \mathrm{kHz}$ ) to accurately propagate the signatures to the ground. We draw the following conclusions from our results:

- The Stardust entry example establishes a benchmark for the level of accuracy attainable by the simulation under the idealized conditions of a known shape and an energy deposition profile dominated by drag. This eliminates fragmentation effects and shape uncertainty from affecting the pressure signature and we obtain excellent agreement between the computed and measured signatures.

- For a single N-wave signature, such as Meteor 20081028, the computation matches the morphology of the measured signature extremely well. The period and amplitude of the computed signature are within $1 \%$ and $10 \%$ of their measured values, respectively, resulting in a sharp estimate of local energy deposition along the meteor trail near the source point.

- More generally, when the source of the signature is predominantly the cylindrical Mach-cone of the ballistic trajectory, the simulation provides an accurate prediction of the leading positive pressure phase of the signature. This includes estimates of the shock rise-time, the zero-peak amplitude and the time to the first zero-crossing of the pressure wave.

- The computational approach independently verifies the predictions of the Ceplecha and ReVelle fragmentation mode ${ }^{27}$ and the density estimates of Ceplecha et al ${ }^{[26}$ for the presented meteor cases.

There are many areas for future work. Extending the method of adjoint weighted residuals to equilibriumgas models would improve the accuracy and efficiency of the nearfield simulation. The use of a computational fluid dynamics solver in the nearfield makes the task of exploring the importance of different shapes and configurations with multiple bodies relatively straightforward. This could potentially improve the prediction of the trailing wave-train, since the single spherical shape used in this study cannot generate multiple compressions and expansions. In addition, the use of solvers appropriate in the transitional flow regime would allow one to lift the 70 kilometer limit on the source height. Roughly half of the 24 signatures analyzed by Silber et al ${ }^{15}$ have source heights above 75 kilometers, so this is an important area of future work.

\section{Acknowledgements}

The authors gratefully acknowledge R. Franz and E. Haering (NASA Armstrong Flight Research Center), and W. Edwards (Natural Resources Canada) for their Stardust pressure signatures. The authors also acknowledge R. Gomez (NASA Johnson Space Center) for providing the surface geometry of the Stardust capsule. This work was supported by the NASA Ames Research Center contract NNA16BD60C through the Science Mission Directorate's Planetary Defense Coordination Office and the Asteroid Threat Assessment Project. P. Brown was supported by grants from the Natural Sciences and Engineering Research Council of Canada, the Canada Research Chairs program and NASA co-operative agreement NNX15AC94A. The 
ELFO infrasound array is funded and operated by Natural Resources Canada. Lorien Wheeler and David Rodriguez provided valuable reviews of this manuscript.

\section{References}

${ }^{1}$ Whipple, F. J. W., "Determination of the Temperature of the Upper Atmosphere by Meteor Observations," Nature, Vol. 112, No. 2821, 1923, pp. 759. doi 10.1038/112759a0

${ }^{2}$ Evers, L. G. and Haak, H. W., "Listening to sounds from an exploding meteor and oceanic waves," Geophys. Res. Lett., Vol. 28, 2001, pp. 41-44. doi 10.1029/2000GL011859

${ }^{3}$ Liszka, L., "Listening to Meteors: Infrasonic Observations of Meteors in Northern Sweden," Scientific report 295, Swedish Institute of Space Physics, SE-981 28 Kiruna, Sweden, April 2008.

${ }^{4}$ Edwards, W. N., "Meteor Generated Infrasound: Theory and Observation," Infrasound Monitoring for Atmospheric Studies, edited by A. Le Pichon, E. Blanc, and A. Hauchecorne, Springer Netherlands, 2009, pp. 361-414. doi 10.1007/978-14020-9508-5

${ }^{5}$ Tsikulin, M. A., "Shock Waves During the Movement of Large Meteorites in the Atmosphere," Technical report, english translation ad 715-537, Naval Intelligence Command, Alexandria, VA, Dec. 1970.

${ }^{6}$ ReVelle, D. O., Acoustics of Meteors - Effects of the Atmospheric Temperature and Wind Structure on the Sounds Produced by Meteors, Ph.D. thesis, University of Michigan, 1974.

${ }^{7}$ ReVelle, D. O., "On Meteor-Generated Infrasound," J. Geophys. Res., Vol. 81, 1976, pp. $1217-1230$. doi 10.1029/JA081i007p01217

${ }^{8}$ Jones, D. L., Goyer, G. G., and Plooster, M. N., "Shock wave from a lightning discharge," J. Geophys. Res., Vol. 73, No. 10, 1968, pp. 3121-3127. doi 10.1029/JB073i010p03121

${ }^{9}$ Few, A. A., "Power spectrum of thunder," J. Geophys. Res., Vol. 74, No. 28, 1969, pp. 6926-6934. doi $10.1029 /$ JC074i028p06926.

${ }^{10}$ ReVelle, D. O. and Whitaker, R. W., "Infrasonic detection of a Leonid bolide: 1998 November 17," Meteoritics \& Planetary Science, Vol. 34, No. 6, 1999, pp. 995-1005. doi 10.1111/j.1945-5100.1999.tb01419.x

${ }^{11}$ Brown, P. G., Edwards, W. N., ReVelle, D. O., and Spurny, P., "Acoustic analysis of shock production by very highaltitude meteors-I: infrasonic observations, dynamics and luminosity," J. Atmos. Sol.-Terr. Phys., Vol. 69, 2007, pp. 600-620. doi $10.1016 /$ j.jastp.2006.10.011

${ }^{12}$ Edwards, W. N., Brown, P. G., Weryk, R. J., and ReVelle, D. O., "Infrasonic observations of meteoroids: Preliminary results from a coordinated optical-radar-infrasound observing campaign," Earth Moon Planet, Vol. 102, 2008, pp. 221-229. doi $10.1007 / \mathrm{s} 11038-007-9154-6$

${ }^{13}$ Silber, E. A. and Brown, P. G., "Optical observations of meteors generating infrasound-I: Acoustic signal identification and phenomenology," J. Atmos. Sol.-Terr. Phys., Vol. 119, 2014, pp. 116-128. doi 10.1016/j.jastp.2014.07.005

${ }^{14}$ Weryk, R. J., Brown, P. G., Domokos, A., Edwards, W. N., Krzeminski, Z., Nudds, S. H., and Welch, D. L., "The Southern Ontario All-sky Meteor Camera Network," Earth, Moon, and Planets, Vol. 102, No. 1, 2008, pp. 241-246. doi 10.1007/s11038007-9183-1

${ }^{15}$ Silber, E. A., Brown, P. G., and Krzeminski, Z., "Optical observations of meteors generating infrasound: Weak shock theory and validation," J. Geophys. Res. Planets, Vol. 120, No. 3, 2015, pp. 413-428. doi 10.1002/2014JE004680

${ }^{16}$ Haynes, C. P. and Millet, C., "A sensitivity analysis of meteoric infrasound," J. Geophys. Res. Planets, Vol. 118, 2013, pp. 2073-2082. doi $10.1002 /$ jgre.20116

${ }^{17}$ Whitham, G. B., "On the propagation of weak shock waves," J. Fluid Mech., Vol. 1, 1956, pp. $290-318$. doi $10.1017 /$ S0022112056000172.

${ }^{18}$ Henneton, M., Gainville, O., and Coulouvrat, F., "Numerical Simulation of Sonic Boom from Hypersonic Meteoroids," AIAA J., Vol. 53, No. 9, 2015, pp. 2560-2570.

${ }^{19}$ Brown, P. G., ReVelle, D. O., Silber, E. A., Edwards, W. N., Arrowsmith, S., Jackson, L. E., Tancredi, G., and Eaton, D., "Analysis of a crater-forming meteorite impact in Peru," J. Geophys. Res., Vol. 113, No. E09007, 2008. doi 10.1029/2008JE003105

${ }^{20}$ Le Pichon, A., Antier, K., Cansi, Y., Hernandez, B., Minaya, E., Burgoa, B., Drob, D., Evers, L. G., and Vaubaillon, J., "Evidence for a meteoritic origin of the September 15, 2007, Carancas crater," Meteoritics \& Planetary Science, Vol. 43, 2008, pp. 1797-1809. doi $10.1111 / \mathrm{j} .1945-5100.2008 . t b 00644 . x$

${ }^{21}$ Aftosmis, M. J. and Nemec, M., "Cart3D Simulations for the First AIAA Sonic Boom Prediction Workshop," AIAA Paper 2014-0558, Jan. 2014. doi 10.2514/6.2014-0558

${ }^{22}$ Plotkin, K., Franz, R. J., and Haering, E. A., "Prediction and Measurement of a Weak Sonic Boom from an Entry Vehicle," Paper 2apa3, Fourth Joint Meeting of the Acoustical Society of America and the Acoustical Society of Japan, Wyle Laboratories and NASA Dryden Flight Research Center, Nov. 2006.

${ }^{23}$ Revelle, D. O. and Edwards, W. N., "Stardust-An artificial, low-velocity "meteor" fall and recovery: 15 January 2006," Meteoritics $\&$ Planetary Science, Vol. 42, No. 2, 2007, pp. 271-299. doi 10.1111/j.1945-5100.2007.tb00232.x

${ }^{24}$ Nemec, M., Aftosmis, M. J., and Brown, P. G., "Numerical prediction of meteoric infrasound signatures," Planetary and Space Science, Vol. 140, June 2017, pp. 11-20. doi 10.1016/j.pss.2017.03.003

${ }^{25}$ Aftosmis, M. J., Nemec, M., Mathias, D. L., and Berger, M. J., "Numerical Simulation of Bolide Entry with Ground Footprint Prediction," 54th AIAA Aerospace Sciences Meeting, AIAA Paper 2016-0998, San Diego, CA, Jan. 2016. doi $10.2514 / 6.2016-0998$

${ }^{26}$ Ceplecha, Z., Borovička, J., Elford, W. G., ReVelle, D. O., Hawkes, R. L., Porubčan, V., and Šimek, M., "Meteor Phenomena and Bodies," Space Science Reviews, Vol. 84, No. 3, 1998, pp. 327-471. doi 10.1023/A:1005069928850. 
${ }^{27}$ Ceplecha, Z. and ReVelle, D. O., "Fragmentation model of meteoroid motion, mass loss, and radiation in the atmosphere," Meteoritics \&S Planetary Science, Vol. 40, No. 1, 2005, pp. 35-54. doi 10.1111/j.1945-5100.2005.tb00363.x

${ }^{28}$ Cheung, S., Edwards, T., and Lawrence, S., "Application of CFD to Sonic Boom Near- and Mid-Field Prediction," 13 th AIAA Aeroacoustics Conference, AIAA Paper 90-3999, Tallahassee, FL, Oct. 1990. doi 10.2514/6.1990-3999

${ }^{29}$ Siclari, M. and Darden, C., "CFD Predictions of the Near-Field Sonic Boom Environment for Two Low Boom HSCT Configurations," 22nd AIAA Fluid Dynamics, Plasma Dynamics 8 Lasers Conference, AIAA Paper 91-1631, Honolulu, HI, June 1991.

${ }^{30}$ Page, J. A. and Plotkin, K. J., "An Efficient Method for Incorporating Computational Fluid Dynamics Into Sonic Boom Prediction," 9th AIAA Applied Aerodynamics Conference, AIAA Paper 91-3275, Baltimore, MD, Sept. 1991.

${ }^{31}$ Maglieri, D. J., Bobbitt, P. J., Plotkin, K. J., Shepherd, K. P., Coen, P. G., and Richwine, D. M., "Sonic Boom: Six Decades of Research," NASA/SP-2014-622, 2014, http://hdl.handle.net/2060/20150006843, visited Sept 2016.

${ }^{32}$ Park, M. A., Aftosmis, M. J., Campbell, R. L., Carter, M. B., Cliff, S. E., and Bangert, L. S., "Summary of the 2008 NASA Fundamental Aeronautics Program Sonic Boom Prediction Workshop," AIAA Paper 2013-0649, Grapevine, TX, Jan. 2013. doi 10.2514/6.2013-649, (51st AIAA Aerospace Sciences Meeting).

${ }^{33}$ Park, M. A. and Morgenstern, J. M., "Summary and Statistical Analysis of the First AIAA Sonic Boom Prediction Workshop," J. of Aircraft, Vol. 53, No. 2, 2016, pp. 578-598. doi 10.2514/1.C033449

${ }^{34}$ Haering, E. A., Murray, J. E., Purifoy, D. D., Graham, D. H., Meredith, K. B., Ashburn, C. E., and Stucky, M., "Airborne Shaped Sonic Boom Demonstration with Computational Fluid Dynamics Comparisons," 43rd AIAA Aerospace Sciences Meeting and Exhibit, AIAA Paper 2005-0009, Reno, NV, Jan. 2005.

${ }^{35}$ Wintzer, M., Ordaz, I., and Fenbert, J. W., "Under-Track CFD-Based Shape Optimization for a Low-Boom Demonstrator Concept," 33rd AIAA Applied Aerodynamics Conference, AIAA Paper 2015-2260, Dallas, TX, June 2015.

${ }^{36}$ Srinivasan, S., Tannehill, J. C., and Weilmuenster, K. J., "Simplified Curve Fits for the Thermodynamic Properties of Equilibrium Air," NASA Reference Publication 1181, National Aeronautics and Space Administration, Aug. 1987.

${ }^{37}$ Aftosmis, M. J., Berger, M. J., and Melton, J. E., "Robust and Efficient Cartesian Mesh Generation for Component-Based Geometry," AIAA J., Vol. 36, No. 6, 1998, pp. 952-960.

${ }^{38}$ Aftosmis, M. J., Berger, M. J., and Adomavicius, G., "A Parallel Multilevel Method for Adaptively Refined Cartesian Grids with Embedded Boundaries," AIAA Paper 2000-0808, Reno, NV, Jan. 2000.

${ }^{39}$ Berger, M. J., Aftosmis, M. J., and Murman, S. M., "Analysis of Slope Limiters on Irregular Grids," AIAA Paper 2005-0490, Reno, NV, Jan. 2005.

${ }^{40}$ Berger, M. J., Aftosmis, M. J., Marshall, D. D., and Murman, S. M., "Performance of a New CFD Flow Solver Using a Hybrid Programming Paradigm," J. of Parallel and Distributed Computing, Vol. 65, 2005, pp. 414-423.

${ }^{41}$ Nemec, M. and Aftosmis, M. J., "Toward Automatic Verification of Goal-Oriented Flow Simulations," NASA/TM 2014218386, National Aeronautics and Space Administration, Center for AeroSpace Information, 7115 Standard Drive, Hanover, MD 21076, 2014, http://hdl.handle.net/2060/20150000864.

${ }^{42}$ Rallabhandi, S. K., "Advanced Sonic Boom Prediction Using the Augmented Burgers Equation," J. Aircraft, Vol. 48, No. 4, 2011, pp. 1245-1253. doi 10.2514/1.C031248

${ }^{43}$ Cleveland, R. O., Propagation of Sonic Booms Through a Real, Stratified Atmosphere, Ph.D. thesis, The University of Texas at Austin, 1995.

${ }^{44}$ Millet, C. and Haynes, C. P., "Stochastic model approach of meteor-generated infrasound," AIAA Paper 2010-7999, Toronto, ON, Aug. 2010.

${ }^{45}$ Yamamoto, M., Hashimoto, A., Aoyama, T., and Sakai, T., "A unified approach to an augmented Burgers equation for the propagation of sonic booms," J. Acoust. Soc. Am., Vol. 137, No. 4, 2015, pp. 1857-1866. doi 10.1121/1.4916833

${ }^{46}$ Sutherland, L. C. and Bass, H. E., "Atmospheric absorption in the atmosphere up to $160 \mathrm{~km}, "$ J. Accoust. Soc. Am., Vol. 115, No. 3, 2004, pp. 1012-1032. doi 10.1121/1.1631937

${ }^{47}$ Anderson, G. R., Aftosmis, M. J., and Nemec, M., "Cart3D Simulations for the Second AIAA Sonic Boom Prediction Workshop," AVIATION 2017, American Institute of Aeronautics and Astronautics (submitted for publication), 2017.

${ }^{48}$ Roache, P. J., "Verification of Codes and Calculations," AIAA Journal, Vol. 36, No. 5, May 1998 , pp. 696-702. doi $10.2514 / 2.457$

${ }^{49}$ Stackpoole, M., Sepka, S., Cozmuta, I., and Kontinos, D., "Post-Flight Evaluation of Stardust Sample Return Capsule Forebody Heatshield Material," AIAA Paper 2008-1202, Toronto, ON, Jan. 2008. doi 10.2514/6.2008-1202

${ }^{50}$ Desai, P. N. and Qualls, G. D., "Stardust Entry Reconstruction," AIAA Paper 2008-1198, Reno, NV, Jan. 2008.

${ }^{51}$ Trumble, K. A., Cozmuta, I., Sepka, S., Jenniskens, P., and Winter, M., "Postflight Aerothermal Analysis of Stardust Sample Return Capsule," J. Spacecraft and Rockets, Vol. 47, No. 5, 2010, pp. 765-774. doi 10.2514/1.41514

${ }^{52}$ Silber, E. A., Observational and theoretical investigations of cylindrical line source blast theory using meteors, $\mathrm{Ph} . \mathrm{D}$. thesis, University of Western Ontario, 2014.

${ }^{53}$ Swinbank, R. and O'Neill, A., "A Stratosphere-Troposphere Data Assimilation System," Monthly Weather Review, Vol. 122, No. 4, 1994, pp. 686-702. doi 10.1175/1520-0493(1994)122<0686:ASTDAS >2.0.CO;2

${ }^{54}$ Hedin, A., Fleming, E., Manson, A., Schmidlin, F., Avery, S., Clark, R., Franke, S., Fraser, G., Tsuda, T., Vial, F., and Vincent, R., "Empirical wind model for the upper, middle and lower atmosphere," J. Atmos. Terr. Phys., Vol. 58, No. 13, 1996, pp. 1421 - 1447. doi 10.1016/0021-9169(95)00122-0.

${ }^{55}$ Picone, J. M., Hedin, A. E., Drob, D. P., and Aikin, A. C., "NRLMSISE-00 empirical model of the atmosphere: Statistical comparisons and scientific issues," J. Geophys. Res., Vol. 107, No. A12, 2002. doi 10.1029/2002JA009430.

${ }^{56}$ American National Standards Institute, "Method for Calculation of the Absorption of Sound by the Atmosphere," ANSI S1.26-1995, Sept. 1995. 